\title{
In or out: Response slowing across housing conditions as a measure of affect in three Western lowland gorillas (Gorilla gorilla gorilla)
}

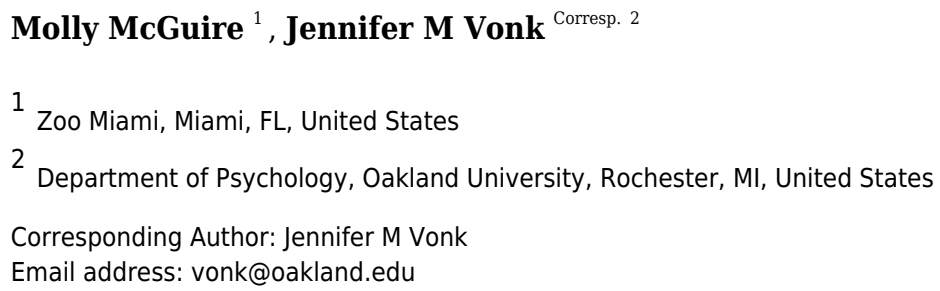

Background: Individuals experiencing negative affect have shown response slowing, a longer latency to respond in relation to baseline, when presented with aversive stimuli. We assessed response slowing in three male gorillas housed in a bachelor group as a function of daytime and nighttime housing arrangements. Methods: In both experiments, three gorillas were rewarded for touching a single image (baseline, non-threatening gorilla or threatening gorilla) on a touchscreen. In Experiment One, they completed 48 50-trial sessions across combinations of three nested daytime and three nighttime conditions. In Experiment Two, they completed eight 50 -trial sessions with novel stimuli across two daytime conditions, which were nested within two nighttime conditions. Housing conditions represented different amounts of space and degree of choice. We predicted that the gorillas would show response slowing to threatening stimuli when space and choice were restricted. Results: We did not observe response slowing in Experiment One, although daytime and nighttime conditions interacted to predict response latencies. The gorillas responded more slowly when they had access to indoors and outdoors overnight compared to when they were in their stalls or together in an indoor habitat, but only if they had been given access to both indoors and outdoors or locked in the indoor habitat the day before. In Experiment Two, the gorillas did show response slowing to threatening stimuli, but this pattern did not interact with housing conditions. Our results, although limited by a small sample, are somewhat consistent with those of a previous study that did not find significant response slowing for apes as a function of aversive testing conditions, although the procedure has been effective in identifying dysregulated fear (high fear in low threat conditions) in macaques. The utility of this paradigm for testing affect in apes awaits further evaluation. 
4

5 In or out: Response slowing across housing conditions as a measure

6 of affect in three Western lowland gorillas (Gorilla gorilla gorilla)

7

16 * Correspondence: Jennifer Vonk, Oakland University, Department of Psychology, 654 Pioneer

17 Drive, Rochester, MI, 48309, USA, vonk@oakland.edu

${ }^{1}$ Zoo Miami, 12400 SW 152nd St, Miami, FL 33177.

${ }^{2}$ Oakland University, Department of Psychology, Rochester, MI, USA. 


\section{Abstract}

21 Background: Individuals experiencing negative affect have shown response slowing, a longer

22 latency to respond in relation to baseline, when presented with aversive stimuli. We assessed

23 response slowing in three male gorillas housed in a bachelor group as a function of daytime and

24 nighttime housing arrangements. Methods: In both experiments, three gorillas were rewarded for

25 touching a single image (baseline, non-threatening gorilla or threatening gorilla) on a

26 touchscreen. In Experiment One, they completed 48 50-trial sessions across combinations of

27 three nested daytime and three nighttime conditions. In Experiment Two, they completed eight

28 50-trial sessions with novel stimuli across two daytime conditions, which were nested within two

29 nighttime conditions. Housing conditions represented different amounts of space and degree of

30 choice. We predicted that the gorillas would show response slowing to threatening stimuli when

31 space and choice were restricted. Results: We did not observe response slowing in Experiment

32 One, although daytime and nighttime conditions interacted to predict response latencies. The

33 gorillas responded more slowly when they had access to indoors and outdoors overnight

34 compared to when they were in their stalls or together in an indoor habitat, but only if they had

35 been given access to both indoors and outdoors or locked in the indoor habitat the day before. In

36 Experiment Two, the gorillas did show response slowing to threatening stimuli, but this pattern

37 did not interact with housing conditions. Our results, although limited by a small sample, are

38 somewhat consistent with those of a previous study that did not find significant response slowing

39 for apes as a function of aversive testing conditions, although the procedure has been effective in

40 identifying dysregulated fear (high fear in low threat conditions) in macaques. The utility of this

41 paradigm for testing affect in apes awaits further evaluation. 


\section{In or out: Response slowing across housing conditions as a measure of affect in Western}

\section{lowland gorillas (Gorilla gorilla gorilla)}

(1)

A recent trend in animal welfare is the assessment of affective states to complement the traditional emphasis on behavior and physiology. Affective states may be the most elusive aspect of welfare to evaluate given that they are not directly observable and any behavioral measures are subject to interpretation. Researchers have increasingly made use of cognitive bias tests, such as judgement bias tests (e.g., Harding, Paul, \& Mendl, 2004; Mendl, Burman, Parker, \& Paul, 2009; Paul, Harding \& Mendl, 2005), to assess when animals are optimistic or pessimistic, but these tests often reveal contradictory patterns of results and may not assess affective states at all (see Perdue, 2017). Furthermore, they often require long periods of training and animals may fail to reach training criteria, making it impossible to conduct the tests that assess affect (e.g., McGuire \& Vonk, 2018; McGuire, Vonk, Fuller \& Allard, 2017a; McGuire, Vonk \& JohnsonUlrich, 2017b) . Despite some question about their interpretation, previous studies have shown that judgement bias tests can be sensitive to affect changes in domestic animals following manipulations of housing conditions (for review see Baciadonna \& McElligott, 2015). We were interested in the affect of three gorillas in a bachelor group as a function of habitat available to them throughout the day and night. Given the challenges of other paradigms used to assess affective state, we adopted a response slowing procedure (Bethell et al., 2016; 2019) both to assess its validity for assessing affect in apes, and for potentially evaluating the effects of housing conditions on the affect of these three gorillas. reaction time to three categories of stimuli (baseline, non-threatening, threatening/aversive), with 
66 the idea that responses to threatening stimuli should be slower than to baseline and non-

67 threatening stimuli, particularly under conditions of threat. Individuals showing this pattern of

68 response are deemed sensitive to threat, which is assumed to be associated with negative affect.

69 The first phase of this method involves a minimal training phase. In Bethell and colleagues'

70 (2016) study, macaques were rewarded for touching a grey square presented on a touchscreen in

71 three spatial locations (left, right, and center). In the testing phase, researchers continued to

72 present the grey squares (to determine a baseline of responding), but also introduced grey

73 squares containing pictures of faces of unfamiliar macaques that were either threatening (direct

74 eye contact) or non-threatening (averted gaze). Macaques that had been subjected to recent

75 veterinary exams, which are considered stressful, displayed a slower latency to touch threatening

76 stimuli compared to baseline. The test was later validated by showing that animals that showed

77 the strongest response to the human intruder task (HIT) - a more invasive assessment of

78 behavioral freezing in response to an unfamiliar human - also showed the greatest behavioral

79 inhibition to the touchscreen task, in that they were most likely to not touch the threatening

80 stimuli (Bethell, Cassidy, Brockhausen, \& Pfefferle, 2019). However, responses in the HIT did

81 not predict response slowing to the aversive stimuli in the computer task.

Although Bethell et al. (2019) did not obtain strong validation for response slowing as a

83 measure of affect, the task is worth investigating further given that it can be implemented quickly

84 without the extended period of training that characterizes most of the cognitive bias assessments

85 available to date. This procedure has also been used with gorillas, chimpanzees and Japanese

86 macaques (Cronin et al., 2018). These primates were tested during an air show over the zoo

87 where they were housed, as well as under quieter conditions. The macaques showed the 
88 predicted response slowing to threatening faces relative to baseline in the noise condition

89 compared to the control conditions, but this pattern was not well evidenced with the apes.

Other researchers have successfully shown that the affect of apes can be captured in a

91 different paradigm using response slowing as a measure. In an emotional Stroop task, human

92 participants are asked to read various words and identify the font color that the words are

93 presented in. The subject of these words may be negative or neutral/positive. People that have

94 been diagnosed with clinical anxiety or depression take longer to identify the color of negative

95 words relative to control subjects (Williams \& Nulty, 1986). Additionally, researchers were able

96 to successfully induce this response slowing by exposing high-anxiety subjects to a mood-

97 manipulation expected to produce a negative mood state (Richards et al., 1992). Adapting this

98 emotional Stroop procedure to chimpanzees, Allritz, Call, and Borkenau (2018) trained

99 chimpanzees to respond to images placed within differently colored borders with one color

100 indicating reward. When negative images, such as images of veterinarians, were placed within

101 the previously rewarded borders, chimpanzees responded more slowly, especially if they had

102 recently experienced an aversive veterinary procedure. This study provided a proof of concept

103 indicating that response times can be used to indicate negative affect in apes. However, Bethell

104 et al. (2019) showed that macaques responded more slowly to pictures of objects than human

105 intruders. This unexpected result, coupled with the fact that the response slowing procedure has

106 not yet been shown to be effective with apes, suggests that the response slowing paradigm

107 requires further validation for use with apes.

108 Previous studies have indicated the effects of different housing conditions on behavior in

109 monkeys (Fontenot, Wiles \& Lynch, 2006) and apes (Hoff, Powell, Lukas \& Maple, 1997). Of

110 relevance to the current study, Aureli and de Waal (1997) examined the behavior of five groups 
111 of captive chimpanzees as a function of whether they had access to indoor and outdoor areas of

112 their habitat or were locked into indoor areas, cutting available space in half. They used

113 behavioral measures as indicators of anxiety and showed that these behaviors were elevated

114 under the higher-density indoor only condition. However, in a later paper, de Waal (1989)

115 introduced the idea of behavioral adjustment to discuss how apes mitigated the presumed

116 negative effects of increased density. Rhesus macaques living in long-term stable groups in

117 captivity showed similar rates of aggression to those living at a field station (Aureli \& de Waal,

118 1997). Others have also found that primates use strategies to reduce stress and aggression when

119 conditions become more crowded (e.g., de Waal, Aureli \& Judge, 2000; Duncan, Jones, Lierop

120 \& Pillay, 2013; Nieuwenhuijsen \& de Waal, 1982). These studies may show that animals

121 mitigate against increased levels of outward aggression through the use of behavioral tactics, but

122 this does not mean that they do not experience higher levels of stress. In fact, de Waal et al.

123 (2000) suggest that efforts to avoid escalations in aggression may come at the cost of increased

124 stress and anxiety. Therefore, it is important to attempt to assess internal affective states in

125 addition to outward behavioral outcomes as a response to variable housing conditions. Like

126 Cronin et al. (2018), we were interested in the effects of environmental changes on a group of

127 captive gorillas, but we assessed effects of housing arrangements.

128 We studied a bachelor group of three relatively young silverback gorillas that experience

129 intermittent aggression due to a shifting dominance hierarchy. These gorillas have access to two

130 different indoor and outdoor habitats at different times of the year. In addition, depending on

131 husbandry and management requirements, the gorillas either spent the night together in the

132 dayroom (the large $205 \mathrm{~m}^{2}$ indoor habitat) or they spent the night individually in a smaller space

$133\left(13 \mathrm{~m}^{2}\right)$. At times, they had access to both indoor and outdoor areas during the day and at night, 
134 resulting in nine different daytime/nighttime housing conditions over the same year long period.

135 We predicted that, during the warmer months, when the gorillas had access to the outdoor

136 habitat, they would display less response slowing due to the positive effects of additional space

137 (i.e., less intense competition for prime space and resources) and increased environmental

138 complexity compared to when they were unable to access this portion of the habitat.

139 Additionally, the gorillas' sleeping arrangements may impact their affect. As adult silverback

140 males, these individuals spend a relatively high proportion of time vying for dominance

141 (personal obs.) and it may be that they experience a tradeoff concerning these two sleep locations

142 (when there is less available space, it is also easier to monitor the location of conspecifics, or,

143 when housed in stalls overnight, the gorillas were isolated and not vulnerable to threat displays

144 from conspecifics). Gorillas may feel more comfortable in one location over the other, which

145 may impact their affect in testing the following day. In addition, given the benefits of choice

146 demonstrated by previous studies of great apes (Kurtycz, Wagner \& Ross, 2014) and bears

147 (Owen, Swaisgood, Czekala \& Lindburg, 2005; Ross, 2016), we expected less response slowing

148 to threatening stimuli when the gorillas had access to both indoor and outdoor spaces (accessed).

149 Gorillas, like macaques, interpret direct eye contact as a threat signal (Schaller, 1963), and thus,

150 might be expected to respond similarly in the task using direct gaze as a threatening cue. Thus,

151 we assessed response slowing by presenting baseline, threatening, and non-threatening stimuli to

152 three male gorillas across these housing conditions in two separate experiments.

\section{Experiment One}

\section{Methods}

Subjects. 
157 "Pende" and Kongo) between the ages of 17-19 years old. The gorillas were housed together at

158 the Detroit Zoo, in Royal Oak, MI. It was believed that Kongo was the dominant male at the time

159 of testing, although Chip had been dominant in the past (keeper observations). Data collection

160 took place in animal holding areas that were inaccessible to the public, three mornings each week

161 at 07:00 h over a period of two months. Gorillas were separated into individual holding areas

162 prior to data collection. These gorillas had previously been trained to use the touch screen for

163 other studies (McGuire \& Vonk, 2018; McGuire, Vonk, Fuller, et al., 2017; McGuire, Vonk, \&

164 Johnson-Ulrich, 2017; Vonk et al., 2014). Training and testing with these animals was approved

165 by the IACUC of Oakland University (12063-R1-A1), and the activities were presented as a

166 form of cognitive enrichment in addition to serving to assess affect.

$167 \quad$ Materials.

168 The experimental apparatus consisted of a durable Panasonic Toughbook CF19 Laptop

169 and 19" VarTech Armorall capacitive touch-screen monitor welded inside a rolling LCD panel

170 cart. Using a $1.2 \mathrm{~m}$ by $1.2 \mathrm{~m}$ plywood ramp, the apparatus was positioned flush against the mesh

171 of the gorilla's holding stalls. The gorillas were given small pieces of chow, various fruits and

172 vegetables from their breakfast trays as a reward for correct responses (in this case, simply

173 touching the stimulus). This task was programmed using Inquisit Version 3 for Windows. All

174 stimuli were presented on a black background. Baseline stimuli were blank grey squares of

175 dimensions 400 by 300 MP Test sessions included both threatening (3) and non-threatening (3)

176 stimuli in addition to the baseline stimuli. To create the three threatening stimuli, photographs of

177 unfamiliar male Western lowland gorillas posturing in an aggressive manner (direct gaze, rigid

178 posture etc.) were placed within the familiar grey baseline square (see Figure 1). To create three 
179 non-threatening stimuli, photographs of unfamiliar males engaged in nonaggressive behavior

180 (eyes averted, relaxed posture etc.) were placed within the familiar grey baseline square (see

181 Figure 1).

$182 \quad$ Procedure.

183 Training. The training phase consisted of ten 15-trial sessions. On each trial, the baseline

184 stimulus was presented at one of three locations (left, center, or right). The stimulus appeared

185 five times at each location in random order. When the gorillas touched this stimulus, a melodic

186 tone was played and they received a single piece of chow or small piece of produce in a PVC

187 feeder affixed to the side of the LCD cart. The next stimulus appeared after a 750 MS ITI

188 immediately after a stimulus was selected.

189 Testing. Each gorilla was presented with 48 testing sessions across all possible housing

190 arrangements. Testing took place between Oct. 2016 and Oct. 2017. The conditions were

191 determined based on where the gorilla had spent the previous day (locked indoors, which

192 consisted of being confined to either the north day room [NDR] or the south day room [SDR],

193 locked outdoors in either the north or south outdoor habitat, or accessed to both indoors and

194 outdoors) and where they had spent the night (locked indoors overnight in either the NDR or

$195 \mathrm{SDR} /$ dayroom or locked in individual stalls or given access to the dayroom and outdoors). We

196 treated indoor and outdoor conditions as separate factors because the issue of space may be

197 qualitatively different when sleeping during the night and when foraging during the day.

198 Both outdoor habitats (seen in Figure 2) included grassy substrates, living, dead and

199 artificial trees and a termite mount. The larger habitat also included a shallow pool. Daytime

200 conditions were nested within nighttime conditions so that we attempted to test each type of

201 daytime condition equally often following each type of nighttime condition, but the conditions 
202 were unbalanced based on important husbandry considerations such as temperature and weather

203 conditions (see Table 1). If the temperature was above $4.4^{\circ} \mathrm{C}$ without heavy precipitation or

204 wind, and barring other maintenance considerations, the gorillas were housed in an outdoor

205 habitat measuring $6131 \mathrm{~m}^{2}$ or $2043 \mathrm{~m}^{2}$. If the temperature was below $1.7^{\circ} \mathrm{C}$, the gorillas were

206 housed in an indoor habitat measuring approximately $205 \mathrm{~m}^{2}$ with a height of $6.7 \mathrm{~m}$ high. They

207 typically had access to both habitats if temperatures were between $1.7-4.4^{\circ} \mathrm{C}$. All areas were

208 equally familiar to the gorillas. Gorillas were housed in the dayroom overnight for three nights

209 and then in two adjoining stalls for one night on a four-night cycle. If the gorillas had spent the

210 night in the dayroom or accessed, they were tested in single adjacent stalls. Stalls measured

211 approximately $2.1 \mathrm{~m}$ wide by $3 \mathrm{~m}$ deep by $2.3 \mathrm{~m}$ high. If they had spent the night in individual

212 stalls, then they were given access to two stalls and thus, they were tested with more space

213 between them. Thus, proximity to conspecifics during testing covaried with nighttime condition.

214 Test sessions consisted of 50 trials and took about ten minutes to complete. These trials

215 consisted of nine threatening stimulus trials, nine non-threatening stimulus trials and 32 baseline

216 trials (see Figure 3). The nine threatening stimulus trials consisted of the three threatening

217 stimuli presented once in each of three locations on the screen (left, center, right). The nine non-

218 threatening stimulus trials consisted of the three non-threatening stimuli presented once in each

219 of three locations on the screen (left, center, right). The 32 baseline trials consisted of the grey

220 square presented in each location 11 times (with one missing trial due to programming error).

221 The first three trials of every session consisted of baseline stimuli presented once at each of the

222 three locations. After the first three trials, the remaining trials were presented in random order

223 with each type of image appearing equally often in each of the three locations on the screen. As 
224 in Training, the gorillas were presented with a piece of chow or small piece of produce randomly

225 for touching the displayed stimulus.

226 Results.

227

228

229

230

231

232

233

234

235

236

237

238

239

240

241

242

243

244

245

246

\section{Analyses.}

Any outliers (trials in which the latency to touch the screen exceeded two standard deviations (SD) above the mean for that type of trial) were removed. Any trials in which the response was faster than $60 \mathrm{~ms}$ were also removed, because this was judged to be the minimum time during which a subject could view the stimulus. A histogram of response latency revealed a positive skew, which was confirmed by skewness statistics reported by SPSS that ranged from 2.16 to 3.38. Kolmogorov-Smirnov tests also revealed that latency data for all subjects violated assumptions of normality with $p$ 's all $<.001$. Thus, latency data was transformed using a $\log _{10}$ transformation. Histograms revealed improved normality following the transformation. A linear mixed effects model was conducted in SPSS 24.0 with subject as a random effect and stimulus type (non-threatening, baseline, and threatening), daytime location (indoor, outdoor, accessed), and overnight location (stall, dayroom and accessed) as fixed effects. We also included all twoway interactions between stimulus type, daytime location and overnight location as well as the three-way interaction.

\section{Effects.}

There was a significant effect of stimulus type $\left(F_{2,6265.01}=3.603, p=.027\right)$, daytime location $\left(F_{2,6265.05}=5.773, p=.003\right)$ and nighttime location $\left(F_{2,6265.09}=54.663, p<.001\right)$ on latency. However, estimates of fixed effects revealed that the gorillas did not touch the threatening stimuli $(M=3.060, S E M=0.037)$ more slowly than the baseline stimuli $(M=3.089$, $S E M=0.036, t=0.79, p=.43)$ or the non-threatening stimuli $(M=3.069, S E M=0.037, t=0.92$, 
$247 p=.93)$. The main effects of daytime and nighttime location were qualified by their significant

248 interaction, $\left(F_{2,6265.04}=21.152, p<.001\right)$, which is depicted in Figure 4. If the gorillas were

249 indoors during the day, there was a significant effect of nighttime location in that latencies to

250 respond after spending the night in their stalls $(M=3.015, S E M=0.040, t=-2.308, p=.021)$

251 and dayroom $(M=2.944, S E M=0.040, t=-4.113, p<.001)$ were faster than latencies when

252 they had access to both indoor and outdoor areas $(M=3.209, S E M=0.048)$. Similarly, if the

253 gorillas had access to both indoors and outdoors during the day, latencies to respond after

254 spending the night in their stalls $(M=3.036, S E M=0.034, t=-2.721, p=.007)$ and dayroom $(M$

$255=3.001, S E M=0.004, t=-4.113, p<.004)$ were faster than latencies when they had access to

256 both indoor and outdoor areas $(M=3.173, S E M=0.038)$. If the gorillas had been outside during

257 the day, then there was no significant difference in latencies between the accessed condition and

258 the other two conditions. The interactions between stimulus type and locations were not

259 significant. Thus, the effects of daytime and nighttime housing conditions were not unique to the 260 threatening stimuli ${ }^{1}$.

\section{Discussion.}

262 Although there was an overall significant effect of stimulus type, it was not in the

263 expected direction of responding more slowly to the threatening stimuli compared to non-

264 threatening and control stimuli. Furthermore, although the gorillas responded with different

265 latencies to the stimuli depending on where they spent the previous day and night, these

266 conditions did not interact with stimulus type. That is, they did not show response slowing to the

267 threatening stimuli in particular. It is reasonable to question whether the lack of effect of housing

268 condition may be due to the fact that housing conditions do not have long lasting effects. For

\footnotetext{
${ }^{1}$ Individual GLMs were also run and the same pattern of daytime by nighttime interactions was observed for each gorilla.
} 
269 example, one might expect stronger effects of currently experienced conditions, compared to

270 conditions that were experienced in the hours (or day) preceding testing. However, the lack of

271 response slowing is consistent with that of Cronin et al. (2018) who did not find significant

272 response slowing for gorillas or chimpanzees during a presumably aversive air show that they

273 were experiencing at the time of testing. In addition, nighttime conditions did impact current

274 housing conditions during testing as the gorillas had additional space between them during

275 testing if they had been housed in the stalls overnight.

276 The pattern of data observed here is also consistent with findings from Bethell et al.

277 (2019) in which macaques showed behavioral inhibition (failure to respond at all to some

278 stimuli) but not response slowing, which might cause one to call the procedure into question.

279 However, it is possible that the lack of effect was due not to a failure of the procedure itself, but

280 of the stimuli we selected. Perhaps the stimuli intended to be threatening or non-threatening were

281 not perceived as such by the gorillas. Furthermore, it is possible that we did not obtain the

282 expected effects of response slowing to threatening stimuli because of the large number of

283 sessions, which may have led to habituation to the threatening stimuli. However, in order to

284 determine whether we might find effects of stimulus type in the early sessions, we ran the

285 models for the first eight sessions alone. We could not include conditions as predictors given the

286 lack of balance of conditions. There was still no main effect of stimulus type.

287 Although we did not obtain significant response slowing to the threatening stimuli, we

288 did observe an interaction of day and night conditions on latencies to respond. The gorillas

289 responded more slowly across stimuli when they had access to both indoor and outdoor areas

290 overnight compared to when they were locked in their stalls or the dayroom, but only if they had

291 been locked indoors or had access to both spaces overnight. When they had spent the day locked 
292 outdoors, there was no effect of nighttime location. Interestingly, this hints at slower responses

293 when coming inside from access to a larger amount of space, complexity and choice of habitat,

294 which are generally viewed as positive conditions (Kurtycz et al., 2014). As it is less clear what

295 general response slowing to all stimuli would mean for determining affect, and given the small

296 sample, it might be misleading to draw conclusions based on these findings.

297 Originally, we had intended to contrast north and south habitats within each of the other

298 conditions as well, thus explaining the large number of sessions and the fact that we could not

299 perfectly balance the nine different conditions within nine-session blocks. The location of the

300 gorillas was not under our control as it was subject to husbandry requirements, weather and

301 temperature. Although access to the outdoor habitats was limited to days when the temperature

302 was above $4.4^{\circ} \mathrm{C}$ such that there were no "outside" or "accessed" daytime conditions in the

303 winter, there were such sessions from early spring to mid fall. There were also warm weather

304 days when the gorillas were locked indoors due to other maintenance requirements, so it is not

305 the case that the conditions were completely confounded with weather, although there was

306 certainly a very close relationship between weather and housing conditions. Thus, it is possible

307 that any condition-related effects were attributable to seasonal or weather effects.

308 Perhaps a more significant limitation is that we did not have access to data regarding the

309 gorillas' behavioral interactions during the time of testing. It is highly likely that agonistic

310 encounters occurring in the time prior to testing had a larger influence on response latencies than

311 the housing conditions did, thus masking effects of our conditions. However, agonistic

312 encounters should not have reduced the likelihood of detecting response slowing to the

313 threatening stimuli in general. 
In an attempt to address the issue of salience and habituation to the photos used in

315 Experiment One, we created a novel set of stimuli (Figure 5) and conducted a reduced number of

316 sessions (8) with these new stimuli in Experiment Two.

\section{Experiment Two}

\section{Method}

The same gorillas participated in Experiment Two using the same apparatus and

procedure. The only difference was that the threatening and non-threatening stimuli were

replaced with novel images also gathered from non-copyrighted images available via the Internet

322 (see Figure 5). In this experiment, we operationalized threatening and non-threatening stimuli

323 more conservatively as direct versus averted gaze (as in Bethell et al., 2016) and used images of

324 only gorilla faces rather than their entire bodies. Moreover, the conditions differed slightly

325 because testing took place from Dec. 2017 to April 2018 so there was no outdoor only condition

326 during the day and no accessed condition overnight. Only eight sessions were conducted to

327 reduce habituation to the photographs. Two sessions were conducted within each of the

328 combination of conditions (daytime; dayroom, access; nighttime; dayroom, stall) in random

329 order based on husbandry constraints. All gorillas participated in all sessions.

\section{Results}

As in Experiment One, latencies more than two SDs above the mean for that stimulus

332 type were removed from the data set. The latency data was again subjected to a $\log _{10}$

333 transformation due to the positive skew. The same linear mixed effects model used in

334 Experiment One was conducted on latency data from Experiment Two. In this model, there was

335 only a significant effect of stimulus type, $\left(F_{2,1177}=3.467, p=.032\right)$. The gorillas responded

336 more slowly to threatening stimuli $(M=3.267, S E M=.074)$ compared to baseline $(M=3.211$, 
$337 S E M=.071)$ and non-threatening stimuli, $(M=3.174, S E M=.074)$, but estimates of fixed

338 effects indicated that these differences were not significant. There were no effects of daytime or

339 nighttime location and no interactions.

\section{Discussion}

341 In this experiment with novel stimuli and fewer sessions, we did observe the expected

342 main effect of stimulus type. The pattern of results revealed response slowing to the threatening

343 stimuli. Thus, it seems important to reduce repeated exposure to the stimuli in order to obtain a

344 response slowing effect. Future studies should ideally introduce novel stimuli in each session. Of

345 course, the results may also indicate a better selection of stimuli in Experiment Two compared to

346 Experiment One. Here, we focused only on whether gorillas were facing forward or had gaze

347 averted rather than making assumptions from posture etc. as to how the images would be 348 perceived by the gorillas.

349 Although these results encourage the use of the response slowing paradigm to some

350 extent, response slowing was not influenced by the housing conditions experienced by the

351 gorillas. This experiment was conducted over a shorter period of time (four months) without the

352 daytime outdoor only and accessed overnight conditions, and it is possible that there was less

353 variability in gorilla affect during this time. Again, we did not have access to observations of

354 their behavior or use of space during this time.

355

356

357

358

359

\section{General Discussion}

Response slowing has been used as a measure of negative affect (fear or anxiety) in both human and nonhuman subjects. For instance, people that displayed Looming Cognitive Style (a tendency to interpret stimuli as threatening or dangerous) also displayed increased reaction times when presented with images of animals, even when those animals were nonthreatening or

Peer] reviewing PDF | (2020:04:47504:2:0:NEW 17 Jun 2020) 
360 appeared to be moving away from the viewer (Riskind, Sagliano, Trojano, \& Conson, 2016).

361 Similar results have been found in nonhuman primate species. For example, Bethell et al. (2016)

362 found that after undergoing veterinary care (assumed to result in negative affect), rhesus

363 macaques displayed a similar trend of response slowing, in this case increasing their reaction

364 times to threatening images of conspecifics making direct eye contact. Cronin et al. (2018) also

365 found that Japanese macaques showed greater response slowing during a loud air show

366 compared to control trials. However, chimpanzees and gorillas tested under similar

367 circumstances showed more subtle effects. As in Experiment One here, it is likely that the apes

368 habituate quickly to the stimuli, indicating the need to present constantly changing stimuli in

369 these designs. Stimuli must also be selected carefully, making dimensions that are important to

370 the study species salient.

371 Somewhat inconsistent support for the response slowing model may be due to the fact

372 that individuals vary in their sensitivity to threat. Bethell and colleagues (2019) found individual

373 differences in response slowing. In their first study, individuals that showed response slowing

374 were those that displayed more significant amounts of freezing in the HIT, verifying that the

375 response slowing may indicate individuals with higher levels of dysregulated fear response.

376 Previous studies have found that dominant animals exhibit higher levels of optimism in

377 judgement bias tasks (tufted capuchin monkeys, Schino, Massimei, Pinzaglia, \& Addessi, 2016;

378 rats, Barker, George, Howarth, \& Whittaker, 2017). Schino et al. (2016) did not find short-term

379 effects of increased optimism after receiving bouts of grooming; however, individuals that

380 overall received more grooming were more optimistic. An understanding of the interaction of

381 social status and response to space could inform management of captive species by helping to

382 predict an animal's behavior and preferences based on their dominance status. Here, we tested 
383 only three gorillas so we are limited in the extent to which we can generalize our findings to

384 other gorillas or other housing conditions. Future studies would benefit from an inclusion of

385 behavioral and perhaps hormonal data along with cognitive testing.

386 We had assumed that the gorillas would feel less stressed and demonstrate less response

387 slowing when provided access to a larger, more complex amount of space, and when given a

388 choice as to which space to occupy. Somewhat surprisingly, we found that gorillas responded

389 more slowly under conditions where they had more space. This result is not altogether

390 unexpected, however. Cordoni and Palagi (2007) found that gorillas did not demonstrate greater

391 conflict in more dense spaces. In fact, they argued that the gorillas adapted their behavior to the

392 increased density by increasing positive behaviors such as touching and reconciliation. These

393 arguments have also been made in studies with rhesus macaques and chimpanzees (de Waal,

394 1989; de Waal et al., 2000; Duncan et al., 2013; Nieuwenhuijsen \& de Waal, 1982). Other

395 studies have also shown that gorillas may be less likely to fully utilize larger habitats compared

396 to even chimpanzees (Ross et al., 2011). Thus, it is possible that they prefer greater proximity

397 and smaller spaces where they can more closely monitor potential threats.

$398 \quad$ There may be other reasons for the complicated relationship between space and

399 aggression in primates (de Waal, 1989; Hosey, 2005). Alexander and Roth (1971) suggested an

400 inverse relationship of environmental space and aggression in Japanese macaques. They found

401 that the macaques displayed increased aggression in response to a reduction in available space,

402 but theorized that this aggression, at least in part, may have also been due to the unfamiliarity of

403 the new space. Similarly, Southwick (1967) demonstrated an inverse relationship between space

404 and aggression in rhesus macaques, yet he also suggested that another factor, social changes,

405 may have had a stronger effect than space alone. In the current study, familiarity and social 
406 structure were not confounds; all of the environmental spaces, regardless of size, were familiar to

407 the gorillas and there were no changes to the social group. The fact that they experienced all

408 conditions frequently on alternating days may help to explain the lack of a consistent effect of

409 housing conditions. In addition, access to outdoor space was confounded with seasonal and

410 temperature changes, which may also correlate with hormonal and dietary changes, such as

411 reduced access to forage during winter months. Fuller and colleagues (2017) found beneficial

412 effects of increased foraging materials in the same group of gorillas tested here. Thus, apparent

413 effects of space may be due to other unconsidered variables instead.

414 It should be noted that although our conditions could generally be taken to represent

415 different amounts of space, the habitats could not solely be reduced to this factor. Outdoor areas

416 also provided different types of enrichment compared to indoor spaces. Different areas vary in

417 their complexity, which may be a predictor of aggression (Hoff et al., 1997). Being housed in

418 stalls versus the dayroom overnight also differed in opportunity to interact and the need to be

419 vigilant to threats from conspecifics. Accessed conditions provided not only more space, but also

420 more choice, which has also been shown to be important to animal welfare (Kurtycz et al., 2014;

421 Ross, 2016). Thus, we believe it would be simplistic to interpret the findings simply in relation to

422 available space. Ideally, a study could control all of the potentially important elements, but that is

423 not typically the case when conducting research outside of the lab. Although the current study

424 has limitations, we believe it contributes to the validation of a promising new assessment tool.

Although not immune to interpretation ambiguities, the response slowing method of

measuring affect may be the best method available for subjects that are ill-suited to the extensive

427 training required for other methods (such as the methods described in previous experiments;

428 McGuire \& Vonk, 2018; McGuire et al., 2017a; 2017b). Because there is minimal training 
429 necessary to implement this measure, it could be a quick and efficient tool for measuring animal

430 affect. With very little training, it was possible to investigate the potential for changes in

431 cognitive bias, as evidenced by response slowing across various seasonal changes in habitat and

432 husbandry routines.

433 It is also possible that, as this measure makes use of an automatic attentional process, that

434 it could be used to identify individuals that may be at risk of developing more problematic

435 symptoms of negative affect, such as stereotypic behaviors, before they develop (Bethell et al.,

436 2016). A similar measure could be used to identify problem animals and intervene through

437 positive changes in the management and husbandry of the animals before a true problem arises.

438 This would be particularly useful for species that are prone to stereotypic behaviors that arise due

439 to stress or poor welfare conditions, which can be notoriously difficult to extinguish once

440 expressed (Mason, 1991).

$441 \quad$ Conclusions

442 The response slowing paradigm will need to be more thoroughly validated before it can

443 be reliably used as a tool for animal welfare researchers through extensive testing and

444 replication, but it is one of the more promising new methods available. One question will be how

445 this paradigm can be adapted to suit species for which computer-based assessments are less

446 feasible, such as equids. One possibility would be to utilize approach times to aversive and

447 preferred feeding locations. Studies have shown that an animal's foraging pattern may be related

448 to their perceptions of safety (Shrader, Brown, Kerley, \& Kotler, 2008; Troxell-Smith, Watters,

449 Whelan, \& Brown, 2017; Troxell-Smith, Whelani, Magle, \& Brown, 2017). The validity of this

450 measure could be determined by looking for other indicators, such as hormonal or behavioral

451 indicators. For instance, the validity of this measure would be supported if hormonal assays 
452 found that hormones related to stress, such as cortisol, or instances of abnormal behaviors, such 453 as hair plucking, were also reduced in response to the gorillas spending time in smaller spaces. In 454 sum, this is a promising method that awaits further validation in a larger number of subjects and 455 species. The jury is still out on its utility for testing apes.

456 


\section{Acknowledgements}

458 We wish to thank the Great Ape keepers and Center for Zoo Animal Welfare at the 459 Detroit Zoo for supporting this research.

$460 \quad$ The authors declare that they have not competing interests.

461 
462

463

464

465

466

467

468

469

470

471

472

473

474

475

476

477

478

479

480

481

482

483

484

485

486

\section{References}

Alexander, B.K., Roth, E.M., 1971. The effects of acute crowding on aggressive behavior of Japanese monkeys. Behaviour, 39, 73-90.

Allritz, M., Call, J., \& Borkenau, P. (2016). How chimpanzees (Pan troglodytes) perform in a modified emotional stroop task. Animal Cognition, 19, 435-449. doi:http://dx.doi.org/10.1007/s10071-015-0944-3

Aureli, F., \& de Waal, Frans B. M. (1997). Inhibition of social behavior in chimpanzees under high-density conditions. American Journal of Primatology, 41, 213-228.

Baciadonna, L., \& McElligott, A. G. (2015). The use of judgement bias to assess welfare in farm livestock. Animal Welfare, 24, 81-91. doi: 10.7120/09627286.24.1.081

Barker, T. H., George, R. P., Howarth, G. S., \& Whittaker, A. L. (2017). Assessment of housing density, space allocation and social hierarchy of laboratory rats on behavioural measures of welfare. PLoS ONE, 12(9), 22.

Bethell, E.J., Cassidy, L.C., Brockhausen, R.R. \& Pfefferle, D. (2019). Toward a standardized test of fearful temperament in primates: A Sensitive alternative to the human intruder task for laboratory-housed Rhesus Macaques (Macaca mulatta). Frontiers in Psychology, 10:1051. doi: 10.3389/fpsyg.2019.01051

Bethell, E., Holmes, A., MacLarnon, A., \& Semple, S. (2016). Emotion evaluation and response slowing in a non-human primate: New directions for cognitive bias measures of animal emotion? Behavioral Sciences, 6(1), 2. http://doi.org/10.3390/bs6010002

Cordoni, G., \& Palagi, E. (2007). Response of captive lowland gorillas (gorilla gorilla gorilla) to different housing conditions: Testing the aggression-density and coping models. Journal of Comparative Psychology, 121, 171-180. doi:http://dx.doi.org/10.1037/0735-7036.121.2.171 
487 Cronin, K. A., Bethell, E. J., Jacobson, S. L., Egelkamp, C., Hopper, L. M., \& Ross, S. R. 488 (2018). Evaluating mood changes in response to anthropogenic noise with a responseslowing task in three species of zoo-housed primates. Animal Behavior and Cognition, 5, 209-221. https://doi.org/10.26451/abc.05.02.03.2018

491 de Waal, F. B. (1989). The myth of a simple relation between space and aggression in captive 492 primates. Zoo Biology, 141-148. https://doi.org/10.1002/zoo.1430080514

de Waal, F. B., Aureli, F., \& Judge, P. G. (2000). Coping with crowding. Scientific American, 494 76-81.

495

496

497

498

499

500

501

502

503

Duncan, L. M., Jones, M. A., van Lierop, M., \& Pillay, N. (2013). Chimpanzees use multiple strategies to limit aggression and stress during spatial density changes. Applied Animal Behaviour Science, 147(1-2), 159-171. doi:http://dx.doi.org.huaryu.kl.oakland.edu/10.1016/j.applanim.2013.06.001

Fontenot, M. B., Wilkes, M. N., \& Lynch, C. S. (2006). Effects of outdoor housing on selfinjurious and stereotypic behavior in adult male rhesus macaques (Macaca mulatta). Journal of the American Association for Laboratory Animal Science, 45, 35-43.

Harding, E.J, Paul, E.S. \& Mendl, M. (2004). Cognitive bias and affective state. Nature, 427, 312.

Hoff, M. P., Powell, D. M., Lukas, K. E., \& Maple, T. L. (1997). Individual and social behavior of lowland gorillas in outdoor exhibits compared with indoor holding areas. Applied Animal Behaviour Science, 54, 359-370. doi:http://dx.doi.org/10.1016/S0168-1591(97)00002-6

Hosey, G. R. (2005). How does the zoo environment affect the behaviour of captive primates? Applied Animal Behaviour Science, 90, 107-129. http://doi.org/10.1016/j.applanim.2004.08.015 
510 Kurtycz, L. M., Wagner, K. E., \& Ross, S. R. (2014). The choice to access outdoor areas affects

511 the behavior of great apes. Journal of Applied Animal Welfare Science, 17, 185-197.

512 doi:http://dx.doi.org/10.1080/10888705.2014.896213

513 Lukas, K. E., Hoff, M. P., \& Maple, T. L. (2003). Gorilla behavior in response to systematic

514 alternation between zoo enclosures. Applied Animal Behaviour Science, 81(4), 367-386.

515 doi:http://dx.doi.org.huaryu.kl.oakland.edu/10.1016/S0168-1591(02)00237-X

516 Mason, G. J. (1991). Stereotypies and suffering. Behavioural Processes, 25, 103-115.

517 http://doi.org/10.1016/0376-6357(91)90013-P

518 McGuire, M. C., \& Vonk, J. (2018). Gorillas (Gorilla gorilla gorilla) fail to learn abstract cues

519 of differential outcomes in a novel cognitive bias test. Animal Behavior and Cognition, 5,

$520 \quad$ 103-117. http://doi.org/10.26451/abc.05.01.08.2018

521 McGuire, M. C., Vonk, J., Fuller, G., \& Allard, S. (2017a). Using an ambiguous cue paradigm to

522 assess cognitive bias in gorillas (Gorilla gorilla gorilla) during a forage manipulation.

523 Animal Behavior and Cognition, 4, 91-104. http://doi.org/10.12966/abc.06.02.2017

524 McGuire, M. C., Vonk, J., \& Johnson-Ulrich, Z. (2017b). Ambiguous results when using the

525 ambiguous-cue paradigm to assess learning and cognitive bias in gorillas and a black bear.

526 Behavioral Sciences, 7(3), 51. http://doi.org/10.3390/bs7030051

527 Mendl, M., Burman, O. H. P., Parker, R. M. A., \& Paul, E. S. (2009). Cognitive bias as an

528 indicator of animal emotion and welfare: Emerging evidence and underlying mechanisms.

529 Applied Animal Behaviour Science, 118, 161-181.

$530 \quad$ http://doi.org/10.1016/j.applanim.2009.02.023

531 Nieuwenhuijsen, K., \& de Waal, F. B. (1982). Effects of spatial crowding on social behavior in a 532 chimpanzee colony. Zoo Biology, 1(1), 5-28. 
doi:http://dx.doi.org.huaryu.kl.oakland.edu/10.1002/zoo.1430010103

534 Paul, E. S., Harding, E. J., \& Mendl, M., (2005). Measuring emotional processes in animals: The 535 utility of a cognitive approach. Neurological and Biobehavioural Reviews, 29, 469-491.

536 http://doi.org/10.1016/j.neubiorev.2005.01.002

537 Perdue, B. M., (2017). Mechanisms underlying cognitive bias in nonhuman primates. Animal

538 Behavior and Cognition, 4, 105-118. http://doi.org/10.12966/abc.08.02.2017

539 Richards, A., French, C. C., Johnson, W., Naparstek, J., \& Williams, J. (1992). Effects of mood

540 manipulation and anxiety on performance of an emotional Stroop task. British Journal of

$541 \quad$ Psychology, 83, 479-491. https://doi.org/10.1111/j.2044-8295.1992.tb02454.x

542 Riskind, J. H., Sagliano, L., Trojano, L., \& Conson, M. (2016). Dysfunctional freezing responses

543 to approaching stimuli in persons with a looming cognitive style for physical

$544 \quad$ threats. Frontiers in Psychology, 7, 521.

545 Ross, S. R. (2006). Issues of choice and control in the behavior of a pair of captive polar bears $546 \quad$ (Ursus maritimus). Behavioural Processes, 73, 117-120.

547 Ross, S. R., Calcutt, S., Schapiro, S. J., \& Hau, J. (2011). Space use selectivity by chimpanzees

548 and gorillas in an indoor-outdoor enclosure. American Journal of Primatology, 73, 197-208.

549 doi:http://dx.doi.org/10.1002/ajp.20891

550 Ross, S. R., Wagner, K. E., Schapiro, S. J., \& Hau, J. (2010). Ape behavior in two alternating

551 environments: Comparing exhibit and short-term holding areas. American Journal of

552 Primatology, 72, 951-959. doi:http://dx.doi.org/10.1002/ajp.20857

553 Schaller, G. E. (1963). The mountain gorilla: ecology and behavior.Oxford, England. Chicago $554 \quad$ Press.

555 Schino, G., Massimei, R., Pinzaglia, M., \& Addessi, E. (2016). Grooming, social rank and 
556 'optimism' in tufted capuchin monkeys: A study of judgement bias. Animal Behaviour, 119,

557 11-16. doi:http://dx.doi.org.huaryu.kl.oakland.edu/10.1016/j.anbehav.2016.06.017

558 Southwick, C.H., 1967. An experimental study of intragroup agonistic behavior in rhesus

559 monkeys (Macaca mulatta). Behaviour, 28, 182-209.

560 Shrader, A. M., Brown, J. S., Kerley, G. I. H., \& Kotler, B. P. (2008). Do free-ranging domestic

561 goats show "landscapes of fear"? Patch use in response to habitat features and predator

$562 \quad$ cues. Journal of Arid Environments, 72, 1811-1819.

$563 \quad$ http://doi.org/10.1016/j.jaridenv.2008.05.004

564 Troxell-Smith, S. M., Watters, J. V., Whelan, C. J., \& Brown, J. S. (2017). Zoo foraging

565 ecology: Preference and welfare assessment of two okapi (Okapia johnstoni) at the

566 Brookfield Zoo. Animal Behavior and Cognition, 4(2), 187-199.

567 https://doi.org/10.12966/abc.05.05.2017

568 Troxell-Smith, S. M., Whelani, C., Magle, S. B., \& Brown, S. (2017). Zoo foraging ecology: 569 development and assessment of a welfare tool for. Animal Welfare, 26, 265-275.

570 Vonk, J., Torgerson-White, L., McGuire, M., Thueme, M., Thomas, J., \& Beran, M. J. (2014).

571 Quantity estimation and comparison in western lowland gorillas (Gorilla gorilla gorilla).

572 Animal Cognition, 17, 755-765. http://doi.org/10.1007/s10071-013-0707-y

573 Williams, J. M. G., \& Nulty, D. D. (1986). Construct accessibility, depression and the emotional 574 stroop task: Transient mood or stable structure? Personality and Individual Differences, 7, 575 485-491. http://doi.org/10.1016/0191-8869(86)90127-3 


\section{Figure Captions}

578 Figure 1. Images used in Experiment 1. A, C and E are threatening images while B, D and F are 579 non-threatening images.

581 Figure 2. An overview of the outdoor gorilla habitats from Google Earth. The South habitat is on 582 the top/left side of the building and the North habitat is on the bottom/right side of the building, 583 which contains the indoor habitats and testing area.

584 Figure 3. Example trials of a) non-threatening, right, b) baseline, center c) threat, left stimuli.

585 Figure 4. Average log transformed latency to touch stimulus (across all stimulus types) as a

586 function of daytime and nighttime conditions in Experiment 1. ${ }^{*} \mathrm{p}=.05, * * \mathrm{p}=.01, * * * \mathrm{p}<.001$.

587 Figure 5. Images used in Experiment 2. A, C and E are direct/threatening images while B, D and $588 \mathrm{~F}$ are averted/non-threatening images.

589 


\section{Table 1 (on next page)}

Number of sessions for each subject in each condition across Experiment 1 
Table 1: Number of sessions for each subject in each condition across Experiment 1.

Daytime

\begin{tabular}{llll}
\hline Nighttime & Indoors & Outdoors & Accessed \\
Stall & 5 & 12 & 4 \\
Dayroom & 4 & 6 & 4 \\
Accessed & 1 & 10 & 2
\end{tabular}

2 
Figure 1

Images used in Experiment 1. A, C and E are threatening images while B, D and $\mathrm{F}$ are non-threatening images. 

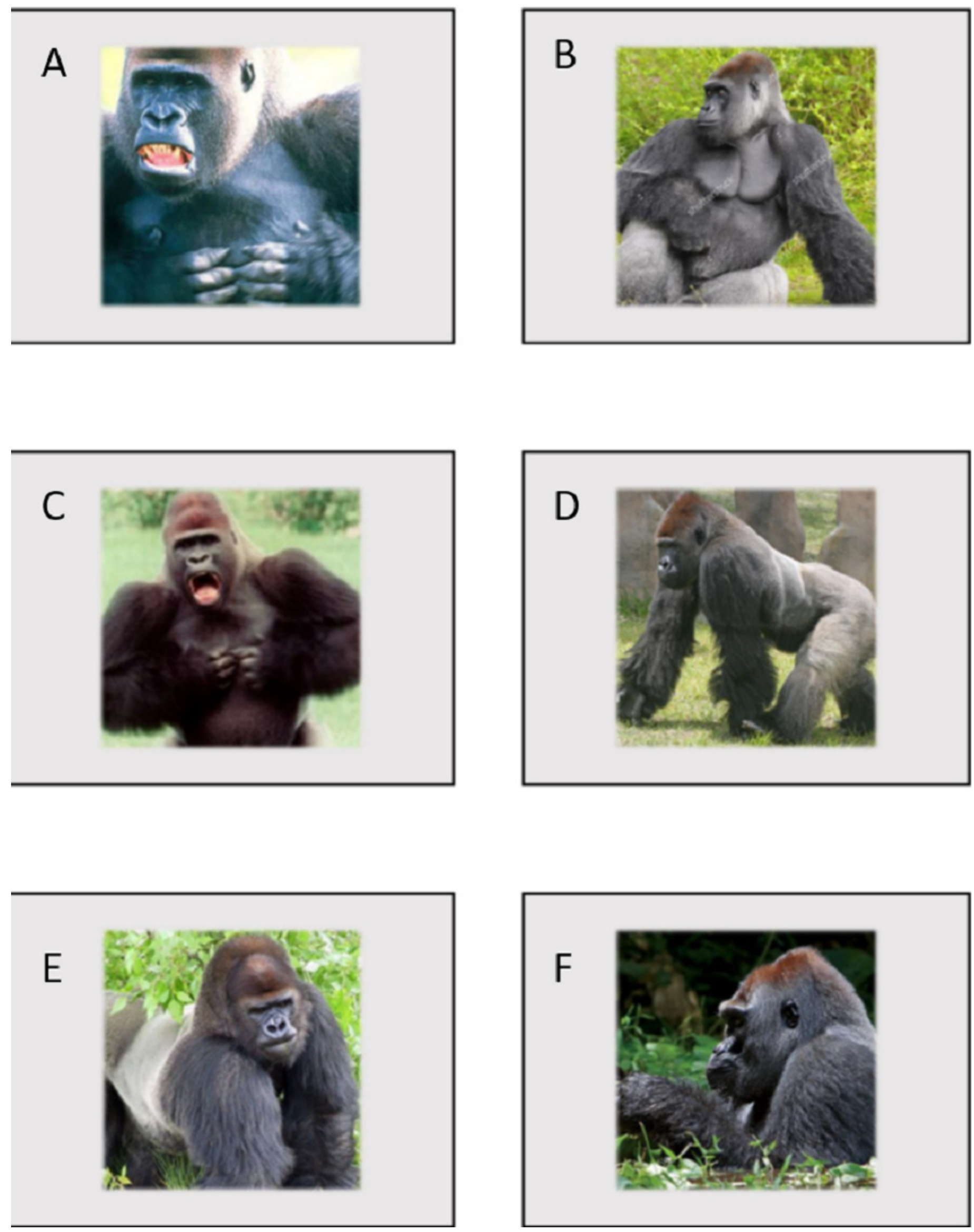
Figure 2

An overview of the outdoor gorilla habitats from Google Earth

The South habitat is on the top/left side of the building and the North habitat is on the bottom/right side of the building, which contains the indoor habitats and testing area (Map Credit: () 2020 Google).

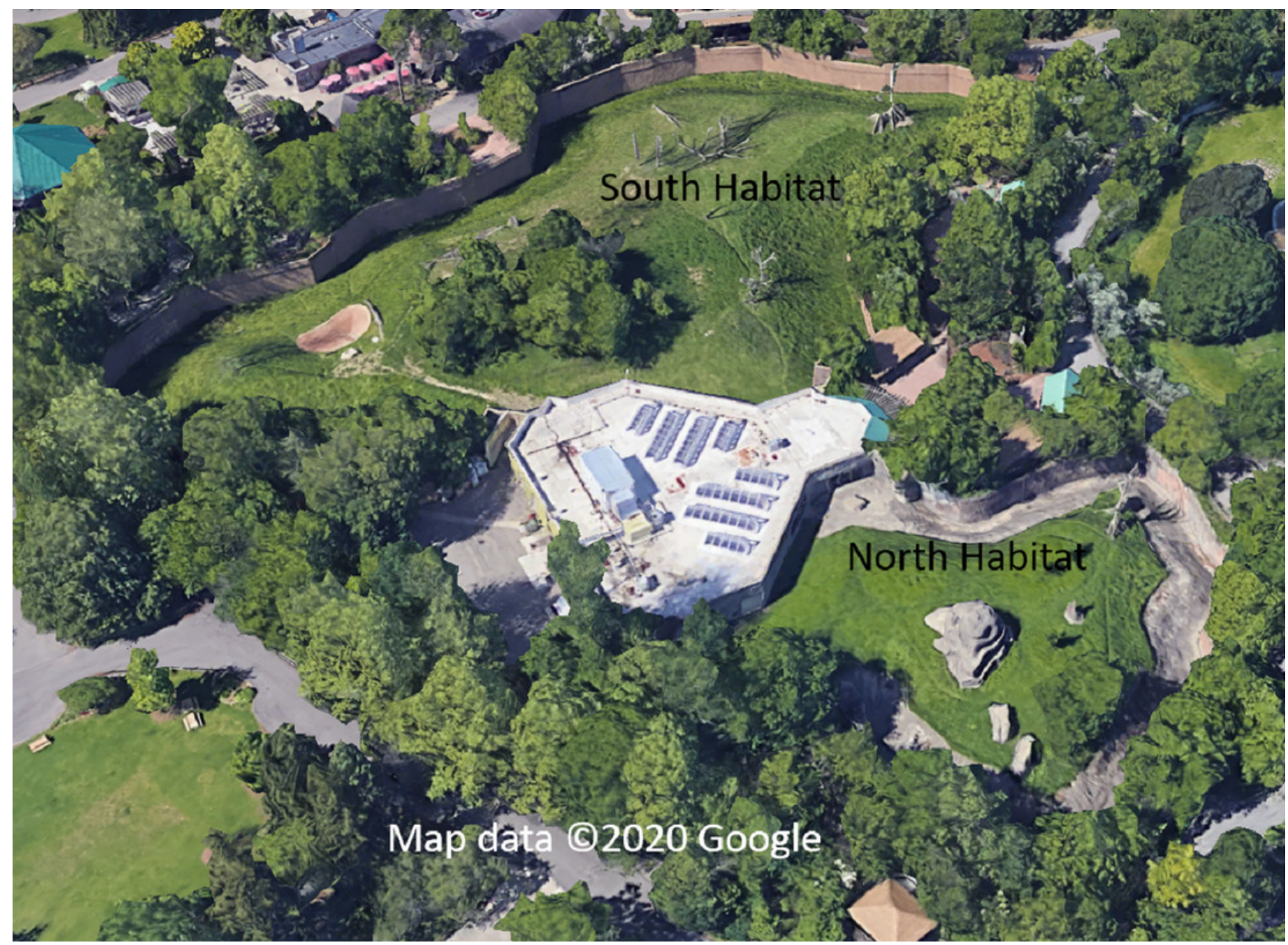


Figure 3

Example test trials.

a) non-threatening, right, b) baseline, center c) threat, left stimuli

a)
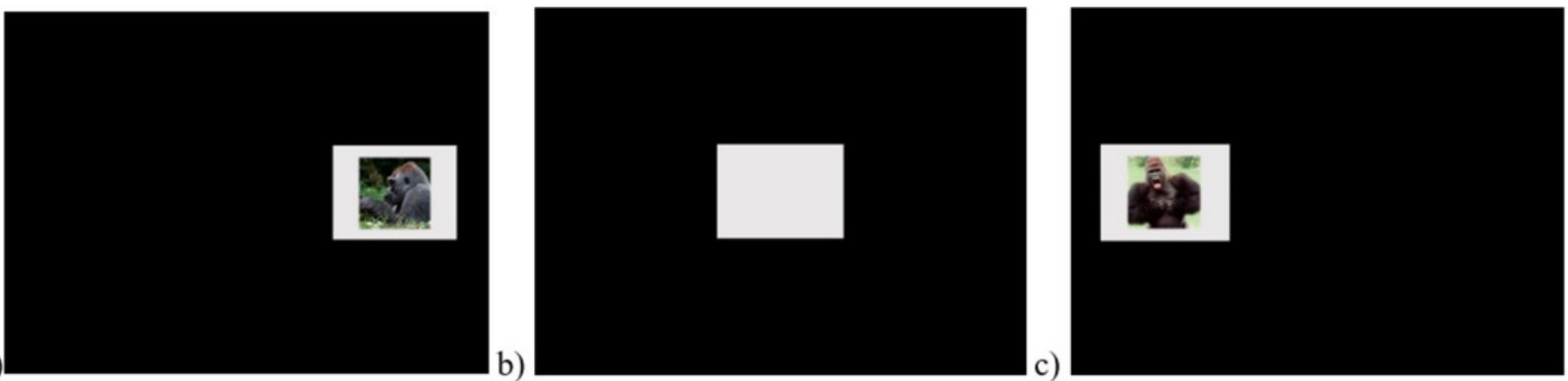
Figure 4

Average log transformed latency to touch stimulus (across all stimulus types) as a function of daytime and nighttime conditions in Experiment 1.

$* p=.05, * * p=.01, * * * p<.001$.

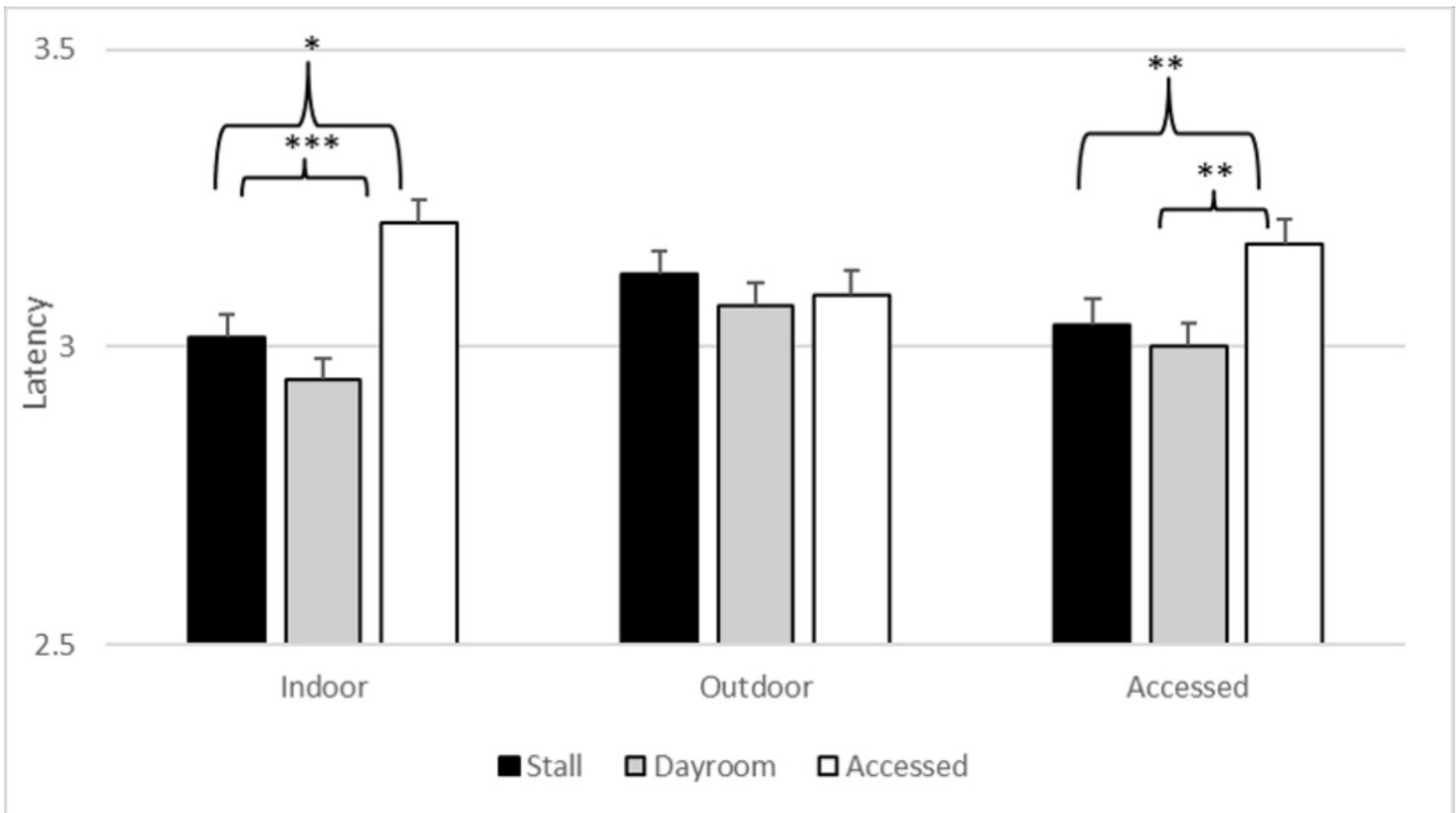


Figure 5

Images used in Experiment 2. $A, C$ and $E$ are direct/threatening images while $B, D$ and $F$ are averted/non-threatening images. 


\section{Direct}

A

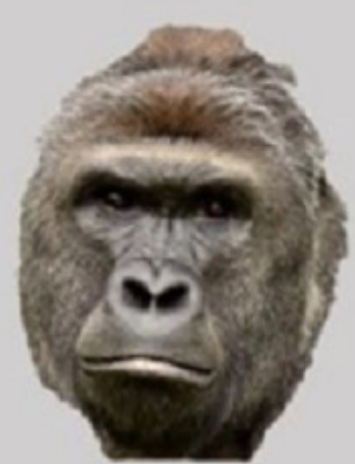

C

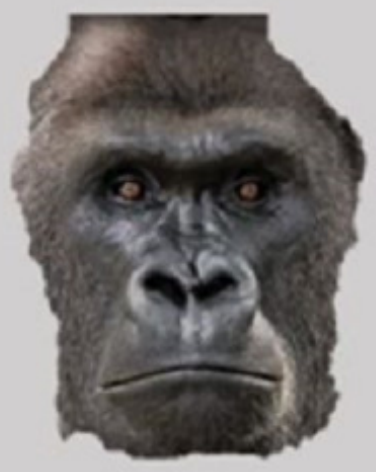

E

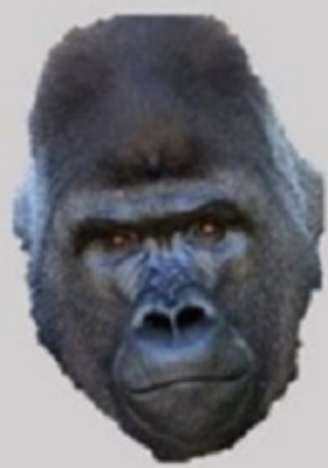

Averted

B

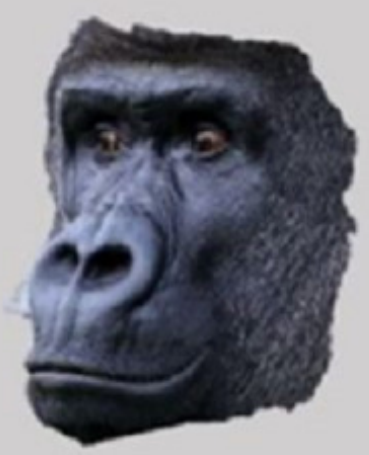

D

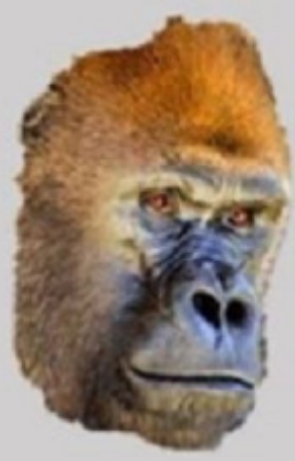

F

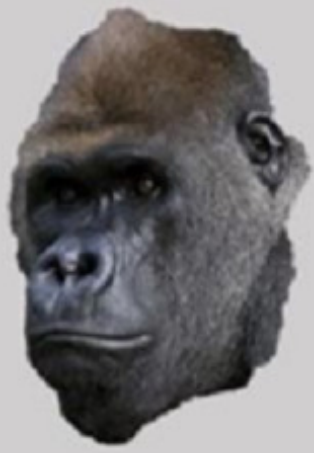

